Review began 12/13/2021 Review ended 12/18/2021 Published 12/29/2021

๑) Copyright 2021

Aljishi et al. This is an open access article distributed under the terms of the Creative Commons Attribution License CC-BY 4.0. which permits unrestricted use, distribution, and reproduction in any medium, provided the original author and source are credited.

\section{Prevalence of Anxiety and Depression in Patients With Multiple Sclerosis in Saudi Arabia: A Cross- Sectional Study}

Rahaif H. Aljishi ${ }^{1}$, Rahaf J. Almatrafi ${ }^{2}$, Zainab A. Alzayer ${ }^{1}$, Bayan A. Alkhamis ${ }^{3}$, Esraa E. Yaseen ${ }^{2}$, Amal M. Alkhotani ${ }^{2}$

1. Department of Medicine, Faculty of Medicine, Hai'l University, Hai'l, SAU 2. Department of Medicine, Faculty of Medicine, Umm Al-Qura University, Makkah, SAU 3. Department of Medicine, Faculty of Medicine, Ibn Sina National College for Medical Studies, Jeddah, SAU

Corresponding author: Rahaif H. Aljishi, rahaif.aljishi@gmail.com

\section{Abstract \\ Introduction}

Multiple sclerosis (MS) is a chronic disease of progressive demyelination in the central nervous system and carries a significant risk for depression and other psychological difficulties associated with low quality of life. There is a paucity of data on the prevalence of anxiety and depression in Saudi Arabia among patients with MS. We conducted a cross-sectional study to determine the prevalence of anxiety and depression in Saudi Arabia among patients with MS by age, disease severity, compliance to medication, and social support.

\section{Methods}

This cross-sectional study measured the prevalence of anxiety and depression in 184 adult patients with MS. The patients were selected through a random sampling method from a pool of MS societies in Saudi Arabia. The participants completed self-administered questionnaires that included demographic variables. The participants also completed the Patient Health Questionnaire-9 (PHQ-9) and the General Anxiety Disorder-7 (GAD-7) questionnaire.

\section{Results}

Depression was detected among 139 (75.5\%) patients with MS, with most participants having mild depression (31\%). More women (83.1\%) experienced depression than men (62.1\%; $\mathrm{p}=0.002)$. Anxiety disorder was present in 123 (66.8\%) patients with MS, and most had mild anxiety $(n=56 ; 30.4 \%)$.

\section{Conclusion}

We found a very high rate of depression and anxiety among patients with MS in Saudi Arabia. Our results highlight the need for periodic screening and examination of patients with MS by psychiatrists to facilitate the early detection and treatment of these comorbidities, potentially improving patient quality of life and health outcomes.

Categories: Internal Medicine, Neurology, Psychiatry

Keywords: saudi arabia, severity, anxiety, depression, multiple sclerosis

\section{Introduction}

Multiple sclerosis (MS) is a chronic disease of progressive demyelination in the central nervous system that shrinks the neuronal sheath and plaque formation in different parts of the brain [1,2]. MS was considered one of the most common neurological disorders and is typically diagnosed in early adulthood [3]. MS occurs in $57-78$ per 100,000 people and affects an estimated 2.5 million people globally [2].

The etiology of MS is still unknown, but several factors most likely contribute to the disease, including genetics (e.g., the presence of the HLA-DRB1 allele), environmental risk factors (e.g., Epstein-Barr virus and low levels of vitamin D), or behavioral factors (e.g., cigarette smoking) [4,5]. The most common symptoms are impaired vision, double vision, limb weakness, gait disability, and bowel/bladder symptoms [6]. Psychological difficulties are a significant concern for patients with MS; most suffer from anxiety, depression, and stress [2]. The lifetime risk of major depression in people with MS is estimated at 50\% compared to the $10 \%-15 \%$ risk of major depression in the general population. Thus, depression plays a critical role in determining the quality of life in patients with MS. When depression is concurrent with anxiety, patients with MS are at an elevated risk of suicide [7].

While still lower than the western and neighboring countries, the prevalence of MS in Saudi Arabia is 40.40 
per 100,000 residents, placing the Kingdom above the low-risk zone per the Kurtzke classification [8]. The prevalence of anxiety and depression in Saudi Arabia among patients with MS has not been well studied $[3,7,9]$. Therefore, we conducted this study to determine the prevalence of anxiety and depression in Saudi Arabia among patients with MS according to age, disease severity, compliance to medication, and social support using a large sample size.

\section{Materials And Methods}

We conducted a cross-sectional study of 184 adult patients affected by MS (66 men and 118 women), selected through a random sampling method from the MS societies in Saudi Arabia. The participants completed selfadministered questionnaires from August 2021 to October 2021. We included patients in Saudi Arabia diagnosed with MS who were older than age 18, and any patients who were not Saudi or did not complete the questionnaire in full were excluded from the study population.

We calculated our sample size using the standard single proportion formula at a confidence level of 95\%, precision of $5 \%$, and significance level of 0.05 , adding $10 \%$ to the original number to compensate for possible losses. Our sample size was limited to 184 due to our criteria for this research, especially since many patients with MS were non-Saudi while our research focused on Saudi individuals only. Data extraction and cleaning of those who did not meet the criteria also resulted in a further reduction in numbers. Before data collection, we obtained Institutional Review Board approval from Umm Al-Qura University Research Ethics Committee in Makkah City, explained the study objectives to the patients, and obtained their voluntary consent before enrolling them in the study.

\section{Questionnaires}

We asked patients to complete an online questionnaire that included demographic variables such as age, sex, marital status, education, and job status. We also collected specific information regarding MS clinical features (e.g., type, onset, duration, pharmacotherapy, and health status compared to the previous year) and other variables, such as comorbidities and physical health.

The patients also completed the Patient Health Questionnaire-9 (PHQ-9) and the General Anxiety Disorder7 (GAD-7) questionnaire. Both questionnaires were translated into Arabic. The PHQ-9 is a short, selfadministered scale based on the nine Diagnostic and Statistical Manual of Mental Disorders-IV criteria for diagnosing depression, with a suggested cutoff score of 10 [10]. The PHQ-9 has a sensitivity of $88 \%$ and specificity of $88 \%$ for severe depression, making it a suitable tool for screening for depression in patients with MS [11]. The GAD-7 is a short, self-administered scale with a cutoff point of 10 that has a sensitivity of $89 \%$ and specificity of $82 \%$ for diagnosing generalized anxiety disorder [12]. The GAD-7 has improved reliability and internal validity in previous studies involving patients with MS [13].

\section{Data analysis}

We used IBM SPSS Statistics for Windows version 22.0. (IBM Corp., Armonk, NY, USA) to analyze the collected data. Two-tailed tests were used for all statistical analyses. A p-value of less than 0.05 was considered statistically significant. The frequency and percent distribution of descriptive analysis was done for all variables, including patients' data, MS medical health conditions, and social support. As for the patient health questionnaire, the discrete scores for different items were summed to achieve an overall score. Based on the questionnaire-reported cutoff points, the overall score was categorized into no/minimal depression, mild depression, moderate depression, moderately severe depression, and severe depression $[10,14]$. Also, the GAD-7 discrete item scores were summed to have an overall score categorized into no/minimal anxiety, mild anxiety, moderate anxiety, and severe anxiety, in reference to the tool-reported cutoff value $[13,15]$. Cross-tabulation was used to assess the distribution of depression and anxiety of patients with MS by their personal and other related data. The significance of relations in cross-tabulation was tested using the Pearson chi-square test and exact probability test for small frequency distributions.

\section{Results}

A total of 184 patients with MS fulfilled the inclusion criteria and participated in the study. The patients ranged from 18 to 59 years old (mean age: $34.9 \pm 11.7$ years). Most participants ( $n=118$; 64\%) were women; 66 were men (35.9\%). Most participants were married, had a university level of education or above, and were unemployed. All patient demographic information and self-perceived mental health data are presented in Table 1. Regarding self-ranking of mental health, 105 (57.1\%) patients thought they were depressed, and 117 (63.6\%) thought they had an anxiety disorder at the time of survey completion. 


\section{Cureus}

Demographic data

Age in years

$18-25$

26-34

$35-43$

$44+$

Gender

Male

Female

Educational level

Secondary/below

University/above

Work

Not working

Full-time work

Part-time work

Free works

Marital status

Single

Married

Divorced/widow

Do you think you depressed now?

Yes

No

Do you think you suffer from an anxiety disorder?

Yes

No
42

54

55

33

66

118

57

127

106

59

9

10

70

98

16

105

79

117

67
$\%$

$22.8 \%$

$29.3 \%$

$29.9 \%$

$17.9 \%$

$35.9 \%$

$64.1 \%$

$31 \%$

$69 \%$

$57.6 \%$

$32.1 \%$

$4.9 \%$

$5.4 \%$

$38 \%$

$53.3 \%$

$8.7 \%$

$57.1 \%$

$42.9 \%$

$63.6 \%$

$36.4 \%$

\section{TABLE 1: Demographic data of patients diagnosed with multiple sclerosis in Saudi Arabia}

Table 2 presents MS-related data among the study participants. While the majority of patients (73.4\%) did not know their type of MS, among those who knew their MS type, the most common was relapsing-remitting MS (RRMS) (15.2\%), followed by progressive-relapsing MS (PRMS) (3.3\%), secondary progressive MS (SPMS) (3.3\%), primary progressive MS (PPMS) (2.7\%), and fulminant type MS (FTMS) (2.2\%). Most patients ( $\mathrm{n}=$ $132 ; 71.7 \%$ ) had been diagnosed over two years before participating in the study, and most $(\mathrm{n}=159 ; 86.4 \%$ ) had been receiving MS treatment. 


\section{Cureus}

Clinical data

Type of multiple sclerosis

Remitting-relapsing MS

Progressive-relapsing MS

Secondary progressive MS

Primary progressive MS

Fulminant type MS

Don't know

Duration of MS

$<2$ years

$>2$ years

Treatment situation

Not on treatment

Still on treatment

How would you rate your health in general?

Poor

Good

Very good

Excellent

How would you rate your health now compared to last year?

Much better than last year

Somewhat better than last year

No change

Somewhat worse

Much worse
$\mathbf{N}$

$\%$

$15.2 \%$

$3.3 \%$

$3.3 \%$

$2.7 \%$

$2.2 \%$

$73.4 \%$

135

52

$28.3 \%$

132

$71.7 \%$

25

$13.6 \%$

159

$86.4 \%$

21

$11.4 \%$

88

$47.8 \%$

48

$26.1 \%$

27

$14.7 \%$

$15.8 \%$

$21.7 \%$

40

$33.7 \%$

$16.3 \%$

$12.5 \%$

TABLE 2: Multiple sclerosis-related data among the study patients in Saudi Arabia

MS: multiple sclerosis

Table 3 presents social support for patients with MS. Only 65 (35.3\%) patients with MS reported social support. Among them, most reported that they had a person who listened to them (89.2\%), gave them love and attention (87.7\%), they trusted and were able to talk to comfortably (86.2\%), prepared them a meal when needed (84.6\%), gave advice (80\%), and take them to doctor's appointments $(83.1 \%)$. 


\section{Cureus}

Social support

Had social support

Yes

No

Do you have someone who(m): $(n=65)$

Helps you when you stay in bed too long?

Take you to the doctor?

Prepares your meals if you cannot make them?

Helps you in your daily affairs if you are ill?

Hears you when you need to talk?

Gives you good advice in difficult times?

Gives you love and attention?

You can listen to?

Gives you information that helps you understand a topic?

You trust and trusts you to talk about themselves and their problems with you?

Hugs you?

You can relax with?

Gives you advice that you value?

Helps you clear your mind?

You share your deepest fears and personal problems?

Guides you on how to solve some of your problems, someone who shares the things that make you happy?

\section{TABLE 3: Social support for patients with multiple sclerosis in Saudi Arabia}

The PHQ-9 results are presented in Table 4. The mean PHO-9 score was 10.5 (range: 0-27). Most patients (83.2\%) reported feeling tired; $70.1 \%$ felt down, depressed, or hopeless; $67.9 \%$ had trouble falling or staying asleep, or sleeping too much; $65.2 \%$ had little interest or pleasure in doing things; $64.7 \%$ had poor appetite or overeating; and $58.7 \%$ had trouble concentrating on activities such as reading the newspaper or watching television. Only $32.1 \%$ thought they would be better off dead or of hurting themselves in some way. 


\section{Cureus}

PHQ-9 items

Little interest or pleasure in doing things

Feeling down, depressed, or hopeless

Trouble falling or staying asleep, or sleeping too much

Feeling tired or having little energy

Poor appetite or overeating

Feeling bad about yourself - or that you are a failure or have let yourself or your family down

Trouble concentrating on things, such as reading the newspaper or watching television

Moving or speaking so slowly that other people could have noticed, or the opposite

- being so fidgety or restless that you have been moving around a lot more than usual

Thoughts that you would be better off dead or of hurting yourself in some way

Overall score (range)

Mean \pm SD

\begin{tabular}{|c|c|c|c|c|c|c|c|}
\hline \multicolumn{2}{|c|}{ Not at all } & \multicolumn{2}{|c|}{$\begin{array}{l}\text { Several } \\
\text { days }\end{array}$} & \multicolumn{2}{|c|}{$\begin{array}{l}\text { More than } \\
\text { half the } \\
\text { days }\end{array}$} & \multicolumn{2}{|c|}{$\begin{array}{l}\text { Nearly } \\
\text { every day }\end{array}$} \\
\hline $\mathrm{N}$ & $\%$ & $\mathrm{~N}$ & $\%$ & $\mathrm{~N}$ & $\%$ & $\mathrm{~N}$ & $\%$ \\
\hline 64 & $34.8 \%$ & 62 & $33.7 \%$ & 22 & $12 \%$ & 36 & $19.6 \%$ \\
\hline 55 & $29.9 \%$ & 59 & $32.1 \%$ & 33 & $17.9 \%$ & 37 & $20.1 \%$ \\
\hline 59 & $32.1 \%$ & 44 & $23.9 \%$ & 23 & $12.5 \%$ & 58 & $31.5 \%$ \\
\hline 31 & $16.8 \%$ & 54 & $29.3 \%$ & 32 & $17.4 \%$ & 67 & $36.4 \%$ \\
\hline 65 & $35.3 \%$ & 57 & $31 \%$ & 26 & $14.1 \%$ & 36 & $19.6 \%$ \\
\hline 79 & $42.9 \%$ & 47 & $25.5 \%$ & 23 & $12.5 \%$ & 35 & $19 \%$ \\
\hline 76 & $41.3 \%$ & 51 & $27.7 \%$ & 19 & $10.3 \%$ & 38 & $20.7 \%$ \\
\hline 102 & $55.4 \%$ & 36 & $19.6 \%$ & 12 & $6.5 \%$ & 34 & $18.5 \%$ \\
\hline 125 & $67.9 \%$ & 21 & $11.4 \%$ & 14 & $7.6 \%$ & 24 & $13 \%$ \\
\hline \multicolumn{8}{|c|}{$0-27$} \\
\hline & & & & & & & \\
\hline
\end{tabular}

TABLE 4: Patient Health Questionnaire-9 item distribution among patients with multiple sclerosis in Saudi Arabia

PHQ-9: Patient Health Questionnaire-9; SD: standard deviation

Depression prevalence and severity data are presented in Figure 1. Depression was detected among 139 (75.5\%) patients with MS, with most participants having mild depression (31\%).

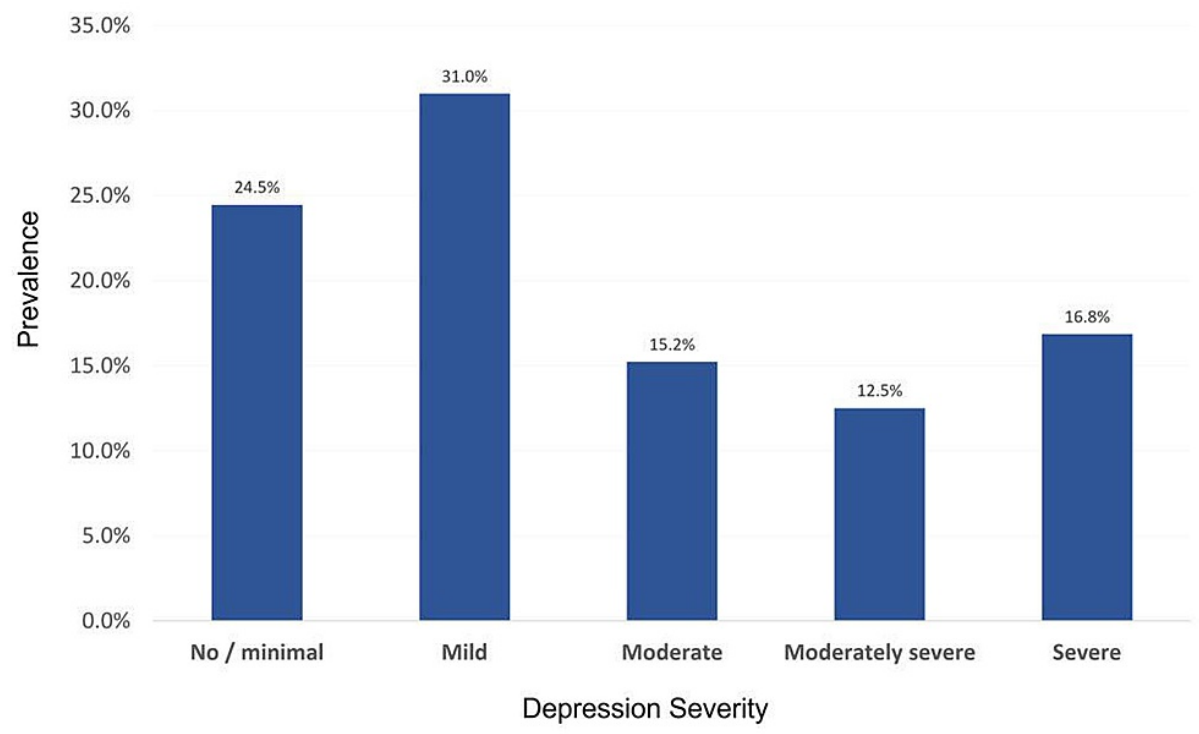

FIGURE 1: Depression severity

Distribution of depression according to severity in patients with multiple sclerosis 


\section{Cureus}

Table 5 presents the GAD- 7 results among our study participants. The mean GAD-7 score for all patients was 8.5 of 21 . Most participants (73.9\%) reported they were easily annoyed or irritable; $67.9 \%$ had trouble relaxing; $65.2 \%$ felt nervous, anxious, or on edge; $64.7 \%$ were unable to stop or control worrying; $64.1 \%$ felt afraid as if something awful might happen; and $52.2 \%$ are so restless that it was challenging to sit still.

\begin{tabular}{|c|c|c|c|c|c|c|c|c|}
\hline \multirow[t]{2}{*}{ GAD-7 items } & \multicolumn{2}{|c|}{ Not at all } & \multicolumn{2}{|c|}{ Several days } & \multicolumn{2}{|c|}{ More than half the days } & \multicolumn{2}{|c|}{ Nearly every day } \\
\hline & $\mathrm{N}$ & $\%$ & $\mathrm{~N}$ & $\%$ & $\mathrm{~N}$ & $\%$ & $\mathrm{~N}$ & $\%$ \\
\hline Feeling nervous, anxious, or on edge & 64 & $34.8 \%$ & 61 & $33.2 \%$ & 23 & $12.5 \%$ & 36 & $19.6 \%$ \\
\hline Not being able to stop or control worrying & 65 & $35.3 \%$ & 64 & $34.8 \%$ & 27 & $14.7 \%$ & 28 & $15.2 \%$ \\
\hline Trouble relaxing & 59 & $32.1 \%$ & 55 & $29.9 \%$ & 28 & $15.2 \%$ & 42 & $22.8 \%$ \\
\hline Being so restless that it's hard to sit still & 88 & $47.8 \%$ & 48 & $26.1 \%$ & 21 & $11.4 \%$ & 27 & $14.7 \%$ \\
\hline Becoming easily annoyed or irritable & 48 & $26.1 \%$ & 64 & $34.8 \%$ & 17 & $9.2 \%$ & 55 & $29.9 \%$ \\
\hline Feeling afraid as if something awful might happen & 66 & $35.9 \%$ & 49 & $26.6 \%$ & 23 & $12.5 \%$ & 46 & $25 \%$ \\
\hline Overall score (range) & \multicolumn{8}{|c|}{$0-21$} \\
\hline Mean \pm SD & \multicolumn{8}{|c|}{$8.5 \pm 6.4$} \\
\hline
\end{tabular}

TABLE 5: General Anxiety Disorder item distribution among patients with multiple sclerosis in Saudi Arabia

SD: standard deviation

Figure 2 presents the prevalence and severity of anxiety among the study participants. Anxiety disorder was present in 123 (66.8\%) patients with MS, and most had mild anxiety ( $\mathrm{n}=56 ; 30.4 \%)$.

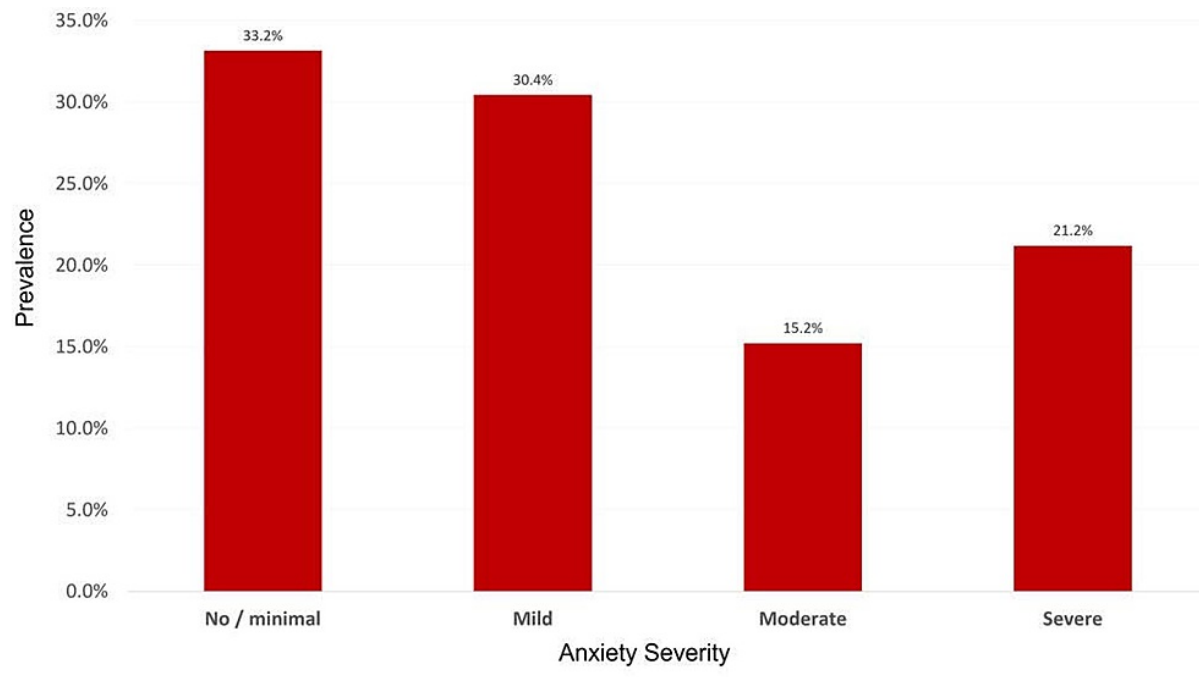

FIGURE 2: Anxiety severity

Distribution of anxiety according to severity in patients with multiple sclerosis

The distribution of depression and anxiety of patients with MS according to demographic data is presented in Table 6. A higher percentage of women $(83.1 \%)$ had depression than men $(62.1 \% ; p=0.002)$. There was a significant association between the type of MS and the prevalence of depression $(0.004)$. There was a significant association between how patients rated their health and the prevalence of depression $(p=0.002)$. Depression was detected among $94.3 \%$ of patients who think they are depressed and $85.5 \%$ of those who think they had an anxiety disorder $(\mathrm{p}=0.001)$. Considering anxiety disorder, $75.4 \%$ of women had anxiety compared with $51.5 \%$ of men $(p=0.001)$. Also, $80.7 \%$ of patients with a secondary level of education showed anxiety disorder compared with $60.6 \%$ of those with a higher educational level $(p=0.007)$. There was a 


\section{Cureus}

significant relationship between the employment status of patients with MS and the prevalence of anxiety among them $(p=0.002)$. There was a significant association between marital status and the prevalence of anxiety among the patients with MS $(\mathrm{p}=0.026)$. There was a significant association between the type of MS and the prevalence of anxiety $(\mathrm{p}=0.001)$. There was a significant association between how patients rated their health and the prevalence of anxiety $(p=0.002)$. There was a significant association between how patients rated their health compared to the previous year and the prevalence of anxiety $(55.2 \% ; \mathrm{p}=0.028)$. Anxiety disorder was detected among $85.7 \%$ of patients who think they are depressed and $80.3 \%$ of those who think they had an anxiety disorder ( $\mathrm{p}=0.001$ for both associations).

\begin{tabular}{|c|c|c|c|c|c|}
\hline \multirow[t]{2}{*}{ Factors } & & \multicolumn{2}{|c|}{ Depressed } & \multicolumn{2}{|c|}{ Anxiety } \\
\hline & & $\mathrm{N}$ & $\%$ & $\mathrm{~N}$ & $\%$ \\
\hline \multirow[t]{4}{*}{ Age in years } & $18-25$ & 35 & $83.3 \%$ & 33 & $78.6 \%$ \\
\hline & $26-34$ & 38 & $70.4 \%$ & 32 & $59.3 \%$ \\
\hline & $35-43$ & 43 & $78.2 \%$ & 37 & $67.3 \%$ \\
\hline & $44+$ & 23 & $69.7 \%$ & 21 & $63.6 \%$ \\
\hline p-value & & \multicolumn{2}{|c|}{0.395} & \multicolumn{2}{|c|}{0.244} \\
\hline \multirow[t]{2}{*}{ Gender } & Male & 41 & $62.1 \%$ & 34 & $51.5 \%$ \\
\hline & Female & 98 & $83.1 \%$ & 89 & $75.4 \%$ \\
\hline p-value & & \multicolumn{2}{|c|}{$0.002^{a}$} & \multicolumn{2}{|c|}{$0.001^{a}$} \\
\hline \multirow[t]{2}{*}{ Educational level } & Secondary/below & 44 & $77.2 \%$ & 46 & $80.7 \%$ \\
\hline & University/above & 95 & $74.8 \%$ & 77 & $60.6 \%$ \\
\hline p-value & & \multicolumn{2}{|c|}{0.727} & \multicolumn{2}{|c|}{$0.007^{a}$} \\
\hline \multirow[t]{4}{*}{ Work } & Not working & 86 & $81.1 \%$ & 80 & $75.5 \%$ \\
\hline & Full-time work & 40 & $67.8 \%$ & 35 & $59.3 \%$ \\
\hline & Part-time work & 7 & $77.8 \%$ & 6 & $66.7 \%$ \\
\hline & Freelancer & 6 & $60 \%$ & 2 & $20 \%$ \\
\hline p-value & & 0.1 & $0.002^{a, b}$ & & \\
\hline \multirow[t]{3}{*}{ Marital status } & Single & 56 & $80 \%$ & 53 & $75.7 \%$ \\
\hline & Married & 71 & $72.4 \%$ & 57 & $58.2 \%$ \\
\hline & Divorced/widow & 12 & $75.0 \%$ & 13 & $81.3 \%$ \\
\hline$p$-value & & \multicolumn{2}{|c|}{0.532} & \multicolumn{2}{|c|}{$0.026^{a}$} \\
\hline \multirow[t]{2}{*}{ Had social support } & Yes & 49 & $75.4 \%$ & 43 & $66.2 \%$ \\
\hline & No & 90 & $75.6 \%$ & 80 & $67.2 \%$ \\
\hline p-value & & \multicolumn{2}{|c|}{0.970} & \multicolumn{2}{|c|}{0.883} \\
\hline \multirow[t]{6}{*}{ Type of multiple sclerosis } & PRMS & 6 & $100 \%$ & 6 & $100 \%$ \\
\hline & RRMS & 27 & $96.4 \%$ & 26 & $92.9 \%$ \\
\hline & PPMS & 4 & $80 \%$ & 5 & $100 \%$ \\
\hline & FTMS & 1 & $25 \%$ & 1 & $25 \%$ \\
\hline & SPMS & 5 & $83.3 \%$ & 2 & $33.3 \%$ \\
\hline & Don't know & 96 & $71.1 \%$ & 83 & $61.5 \%$ \\
\hline$p$-value & & \multicolumn{2}{|c|}{$0.004^{a, b}$} & \multicolumn{2}{|c|}{$0.001^{a, b}$} \\
\hline Duration of MS & $<2$ years & 37 & $71.2 \%$ & 37 & $71.2 \%$ \\
\hline
\end{tabular}




\section{Cureus}

\begin{tabular}{|c|c|c|c|c|c|}
\hline & $>2$ years & 102 & $77.3 \%$ & 86 & $65.2 \%$ \\
\hline $\mathrm{p}$-value & & \multicolumn{2}{|l|}{0.385} & \multicolumn{2}{|c|}{0.436} \\
\hline \multirow[t]{2}{*}{ Treatment situation } & Not on treatment & 19 & $76 \%$ & 19 & $76 \%$ \\
\hline & Still on treatment & 120 & $75.5 \%$ & 104 & $65.4 \%$ \\
\hline $\mathrm{p}$-value & & \multicolumn{2}{|l|}{0.954} & \multicolumn{2}{|c|}{0.296} \\
\hline \multirow[t]{4}{*}{ How would you rate your health in general? } & Poor & 18 & $85.7 \%$ & 18 & $85.7 \%$ \\
\hline & Good & 75 & $85.2 \%$ & 65 & $73.9 \%$ \\
\hline & Very good & 31 & $64.6 \%$ & 29 & $60.4 \%$ \\
\hline & Excellent & 15 & $55.6 \%$ & 11 & $40.7 \%$ \\
\hline $\mathrm{p}$-value & & \multicolumn{2}{|l|}{$0.002^{\mathrm{a}}$} & \multicolumn{2}{|c|}{$0.002^{a}$} \\
\hline \multirow{5}{*}{ How would you rate your health now compared to last year? } & Much better than last year & 19 & $65.5 \%$ & 16 & $55.2 \%$ \\
\hline & Somewhat better than last year & 32 & $80 \%$ & 29 & $72.5 \%$ \\
\hline & No change & 43 & $69.4 \%$ & 35 & $56.5 \%$ \\
\hline & Somewhat worse & 26 & $86.7 \%$ & 23 & $76.7 \%$ \\
\hline & Much worse & 19 & $82.6 \%$ & 20 & $87 \%$ \\
\hline $\mathrm{p}$-value & & \multicolumn{2}{|l|}{0.205} & \multicolumn{2}{|c|}{$0.028^{\mathrm{a}}$} \\
\hline \multirow[t]{2}{*}{ Do you think you are depressed now? } & Yes & 99 & $94.3 \%$ & 90 & $85.7 \%$ \\
\hline & No & 40 & $50.6 \%$ & 33 & $41.8 \%$ \\
\hline $\mathrm{p}$-value & & \multicolumn{2}{|l|}{$0.001^{a}$} & \multicolumn{2}{|c|}{$0.001^{a}$} \\
\hline \multirow[t]{2}{*}{ Do you think you suffer from an anxiety disorder? } & Yes & 100 & $85.5 \%$ & 94 & $80.3 \%$ \\
\hline & No & 39 & $58.2 \%$ & 29 & $43.3 \%$ \\
\hline $\mathrm{p}$-value & & \multicolumn{2}{|l|}{$0.001^{\mathrm{a}}$} & \multicolumn{2}{|c|}{$0.001^{\mathrm{a}}$} \\
\hline
\end{tabular}

\section{TABLE 6: Distribution of depression and anxiety of patients with MS by their demographic data}

MS: multiple sclerosis; PRMS: progressive-relapsing MS; RRMS: remitting-relapsing MS; PPMS: primary progressive MS; FTMS: fulminant type MS; SPMS: secondary progressive MS

aStatistically significant $(p<0.05)$

${ }^{\mathrm{b}}$ Exact probability test

\section{Discussion}

Specific sociodemographic characteristics, such as being female, stresses, parental depression, and specific features, behavior patterns, and dispositions are all contributing causes for depression. These risk factors are linked to biological and genetic causes [16]. As with depression, most anxiety disorders affect women more than men. Anxiety disorders are often accompanied by major depression, alcohol and other substance abuse problems, and personality disorders [17].

Effective therapies for MS can result in fewer depression symptoms, improved psychosocial functioning, and improved quality of life [18]. Therefore, measuring the quality of life in patients with depression is essential when assessing MS therapy outcomes [19].

The high prevalence of psychiatric disorders such as anxiety and depression in the MS population raises a question about the relationship between these conditions. A recent cross-sectional study in Saudi Arabia found that $89.9 \%$ of patients with MS suffered from mild to severe depressive symptoms. There was a high risk among unemployed patients (37.39\%), and the severity of depression was positively related to education level [3]. Another study done in the United Arab Emirates revealed that 17.6\% of patients with MS reported a 
PHQ-9 score compatible with the diagnosis of depressive disorder, and $20 \%$ had a GAD-7 score compatible with anxiety disorder. The study showed no statistical difference in the risk of developed depression or anxiety disorders between the MS and general populations [7]. A study conducted in the United Kingdom using the Hospital Anxiety and Depression Score showed that $54.1 \%$ of patients with MS had anxiety, and 46.9\% had depression. Patients with SPMS had more severe depression than other MS types [9].

In our study, most participants were female (69\%) and aged 35-43 (29.9\%) or 26-34 (29.3\%). Another study found that women were twice as likely to develop MS than men in general [20], which explains the high female-to-male ratio in our study population. A previous study reported that MS occurred more commonly in patients aged 20-40, which aligns with our study population age distribution [2]. However, another study reported significant variability in the age of diagnosis for patients with MS [20]. A lack of awareness of MS is a widespread issue across Saudi Arabia, as noted in our study and previous studies [21,22]. A minority of participants in our study knew the type of MS they had. In our study, 3.3\% of patients with MS had SPMS and $3.3 \%$ had PPMS. A previous study found that $12.1 \%$ of patients with MS had SPMS, but only $2.6 \%$ had PPMS [23]. The likelihood of RRMS turning to SPMS can be reduced with early diagnosis and treatment [23].

We used the PHQ-9 to measure the occurrence and level of depression among our participants. We found that $24.5 \%$ of the participants had no or minimal depression, 31\% had mild depression, $15.2 \%$ had moderate depression, and $12.5 \%$ and $16.8 \%$ had moderately severe and severe depression, respectively. A study conducted in Saudi Arabia found that $24.8 \%$ suffered from mild depression, $23.9 \%$ had moderate depression, $22.3 \%$ had moderately severe, and $18.9 \%$ had severe depression [3]. Biodemographic and environmental differences might explain the variation in the severity of depression and MS types among patients.

Depression peaked in patients aged 18-24 (83.3\%), whereas a study in Riyadh found that this same age group had lower percentages of depression ranging from $9.26 \%$ to $33.33 \%$, depending on depression severity, but moderate depression was the most common [24]. The participants in our study age 44 or older had more prevalent depression (69.7\%) than the findings from a previous cross-sectional study that reported depression in $18.42 \%-28.95 \%$ of patients, depending on depression severity [24].

Anxiety also peaked in the 18-24-year-old group, with a prevalence of 78.6\%. Another study found that $23.0 \%$ of patients with MS had severe stress, and $44.8 \%$ had moderate stress without specifying age groups [2]. One study found that anxiety rates peaked in people older than 60 with up to $50 \%$ prevalence for both mild and moderate anxiety [24]. Women with MS were more likely to have depression (83.1\%) and anxiety (75.4\%) than men, which aligns with two previous studies [2,3]. We found that education level and work status affected depression and anxiety among patients with MS, which supports the findings of a previous study that reported that patients in a good economic state had lower rates of depression and anxiety [2].

In our study, depression was more prevalent among unmarried participants, which contrasts with reports from a study conducted in Iran that reported higher levels of depression in married patients with MS. The effect of marital status may depend on location, community, and culture [2]. Social support did not seem to significantly affect the depression or anxiety rate in our study population.

PRMS had a 100\% depression and 100\% anxiety rate, indicating that PRMS significantly impacts patients' quality of life. Also, we noted that patients who had lived with MS for more than two years were more likely to be depressed, but patients who were more recently diagnosed with MS had higher rates of anxiety. Treatment had a positive effect on anxiety among patients with MS. This trend points to the need for better educational health systems that will help and encourage patients to develop better coping mechanisms. Given that most study participants reported their health as worsening and many felt depressed or anxious, psychological interventions and screenings must be incorporated into MS treatment protocols.

\section{Limitations and strengths}

Our study was limited by the sample size, which restricted data interpretation. Our study was also limited in that we used a self-reported questionnaire to collect data, which may introduce bias and affect the validity of our results. Furthermore, most participants did not know what type of MS disorder they had, which could have affected our results in the correlation between the type of the disease and the anxiety or depression level. Despite the limitations, our study is of public health importance in exploring the quality of care delivered to patients with MS in Saudi Arabia.

\section{Conclusions}

We found a very high rate of depression and anxiety among patients with MS, which highlights the need for periodic screening and examination by psychiatrists to facilitate the early detection and treatment of these comorbidities. Patients with PRMS are at greater risk of developing depression and anxiety than patients with other types of MS. Therefore, patients with PRMS require special attention and additional interventional measures. Patient education programs are essential for mitigating anxiety and depression and addressing quality of life concerns. Further investigations are warranted to determine the impact of depression and anxiety on disease progression and outcomes. Moreover, additional studies are needed to explore the pathological association between MS and psychological disorders. 


\section{Additional Information \\ Disclosures}

Human subjects: Consent was obtained or waived by all participants in this study. The Biomedical Research Ethics Committee issued approval HAPO-02-K-012-2021-08-715. Proposal Title (in English): Prevalence of Anxiety and Depression in Patients With Multiple Sclerosis in Saudi Arabia The Biomedical Research Ethics Committee has evaluated and examined the abovementioned research proposal and has found it to be in accordance with the specifications and conditions of the ethics of scientific research. The Committee has accordingly granted the Principal Investigator final approval concerning the ethics of scientific research. The Principle Investigator is permitted to initiate the implementation of scientific research procedures within faculty facilities and laboratories, in addition to the regional research centers and hospitals, and publish in scientific journals. The Principal Investigator must provide a written statement to the Vice President of postgraduate studies and scientific research regarding any changes in the research plan, and the committee shall decide whether a new approval is needed or not. Animal subjects: All authors have confirmed that this study did not involve animal subjects or tissue. Conflicts of interest: In compliance with the ICMJE uniform disclosure form, all authors declare the following: Payment/services info: All authors have declared that no financial support was received from any organization for the submitted work. Financial relationships: All authors have declared that they have no financial relationships at present or within the previous three years with any organizations that might have an interest in the submitted work. Other relationships: All authors have declared that there are no other relationships or activities that could appear to have influenced the submitted work.

\section{Acknowledgements}

We thank Dr. Mamdoh Kalakatawy of Al Umm Al-Qura University for his help in the data collection process for this study. This study was partially supported by the Azm Multiple Sclerosis Society and Arfa Multiple Sclerosis Society by helping us contact patients.

\section{References}

1. Alhazzani AA, Alqahtani MS, Alahmari MS, et al.: Quality of life assessment among multiple sclerosis patients in Saudi Arabia. Neurosciences (Riyadh). 2018, 23:140-7. 10.17712/nsj.2018.2.20170335

2. Karimi S, Andayeshgar B, Khatony A: Prevalence of anxiety, depression, and stress in patients with multiple sclerosis in Kermanshah-Iran: a cross-sectional study. BMC Psychiatry. 2020, 20:166. 10.1186/s12888-02002579-Z

3. Alhussain H, Aldayel AA, Alenazi A, Alowain F: Multiple sclerosis patients in Saudi Arabia: prevalence of depression and its extent of severity. Cureus. 2020, 12:e7005. 10.7759/cureus.7005

4. Nischwitz S, Müller-Myhsok B, Weber F: Risk conferring genes in multiple sclerosis . FEBS Lett. 2011, 585:3789-97. 10.1016/j.febslet.2011.03.037

5. O'Gorman C, Lucas R, Taylor B: Environmental risk factors for multiple sclerosis: a review with a focus on molecular mechanisms. Int J Mol Sci. 2012, 13:11718-52. 10.3390/ijms130911718

6. Kister I, Bacon TE, Chamot E, Salter AR, Cutter GR, Kalina JT, Herbert J: Natural history of multiple sclerosis symptoms. Int J MS Care. 2013, 15:146-58. 10.7224/1537-2073.2012-053

7. Alsaadi T, El Hammasi K, Shahrour TM, et al.: Prevalence of depression and anxiety among patients with multiple sclerosis attending the MS Clinic at Sheikh Khalifa Medical City, UAE: Cross-Sectional Study. Mult Scler Int. 2015, 2015:487159. 10.1155/2015/487159

8. AlJumah M, Bunyan R, Al Otaibi H, et al.: Rising prevalence of multiple sclerosis in Saudi Arabia, a descriptive study. BMC Neurol. 2020, 20:49. 10.1186/s12883-020-1629-3

9. Jones KH, Ford DV, Jones PA, et al.: A large-scale study of anxiety and depression in people with Multiple Sclerosis: a survey via the web portal of the UK MS Register. PLoS One. 2012, 7:e41910. 10.1371/journal.pone.0041910

10. Kroenke K, Spitzer RL, Williams JB: The PHQ-9: validity of a brief depression severity measure . J Gen Intern Med. 2001, 16:606-13. 10.1046/j.1525-1497.2001.016009606.x

11. Patrick S, Connick P: Psychometric properties of the PHQ-9 depression scale in people with multiple sclerosis: a systematic review. PLoS One. 2019, 14:e0197943. 10.1371/journal.pone.0197943

12. Spitzer RL, Kroenke K, Williams JB, Löwe B: A brief measure for assessing generalized anxiety disorder: the GAD-7. Arch Intern Med. 2006, 166:1092-7. 10.1001/archinte.166.10.1092

13. Löwe B, Decker O, Müller S, Brähler E, Schellberg D, Herzog W, Herzberg PY: Validation and standardization of the Generalized Anxiety Disorder Screener (GAD-7) in the general population. Med Care. 2008, 46:266-74. 10.1097/MLR.0b013e318160d093

14. Patient Health Questionnaire-9 (PHQ-9) . (2021). Accessed: December 2, 2021: https://www.hiv.uw.edu/page/mental-health-screening/phq-9.

15. Generalised Anxiety Disorder Assessment (GAD-7). (2021). Accessed: December 2, 2021: https://www.corc.uk.net/outcome-experience-measures/generalised-anxiety-disorder-assessment-gad-7/.

16. Hammen C: Risk factors for depression: an autobiographical review . Annu Rev Clin Psychol. 2018, 14:1-28. 10.1146/annurev-clinpsy-050817-084811

17. Craske MG, Stein MB: Anxiety. Lancet. 2016, 388:3048-59. 10.1016/S0140-6736(16)30381-6

18. Hofmann SG, Curtiss J, Carpenter JK, Kind S: Effect of treatments for depression on quality of life: a metaanalysis. Cogn Behav Ther. 2017, 46:265-86. 10.1080/16506073.2017.1304445

19. Kennedy SH, Eisfeld BS, Cooke RG: Quality of life: an important dimension in assessing the treatment of depression?. J Psychiatry Neurosci. 2001, 26:S23-8.

20. Walton C, King R, Rechtman L, et al.: Rising prevalence of multiple sclerosis worldwide: Insights from the 


\section{Cureus}

Atlas of MS, third edition. Mult Scler. 2020, 26:1816-21. 10.1177/1352458520970841

21. Amer MG, AlZahrani WA, AlZahrani AA, Altalhi FA, Alrubaie SS, Alsini RA: Assessment of multiple sclerosis awareness: knowledge and attitude among Saudi population in Taif city, KSA. Int J Adv Res. 2016, 4:1758-

66.

22. Hudaif HSA, Bwardi NA, Kojan S: P049 - Assessment of multiple sclerosis awareness and knowledge among the Saudi population in Riyadh City. Mult Scler Relat Disord. 2014, 3:758. 10.1016/j.msard.2014.09.198

23. Nazish S, Shahid R, Zafar A, et al.: Clinical presentations and phenotypic spectrum of multiple sclerosis at a University Hospital in Saudi Arabia. J Clin Neurol. 2018, 14:359-65. 10.3988/jcn.2018.14.3.359

24. Bahathig A, Alblowi MA, Alhilali AA, et al.: The prevalence and association of depression and anxiety with multiple sclerosis in Riyadh, Saudi Arabia: a cross-sectional study. Cureus. 2020, 12:e12389.

10.7759/cureus.12389 